\title{
Material removal, correction and laboratory X-ray diffraction
}

\author{
Eric Wasniewski ${ }^{1, a *}$, Baptiste Honnart ${ }^{1}$, Fabien Lefebvre ${ }^{1, b}$, Eric Usmial ${ }^{1, c}$ \\ ${ }^{1}$ CETIM, laboratoire d'analyses physiques, 52 avenue Felix Louat 60300 Senlis, France

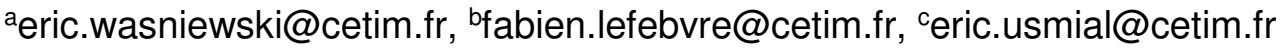

Keywords: Material removal; Laboratory X-ray diffraction ; correction

\begin{abstract}
Laboratory X-ray diffraction is commonly used for surface residual stresses determination. Nevertheless, the in-depth residual stress gradient also needs to be known. Chemical or electro-polishing method is generally used for material removal. However, material removal may seek a new equilibrium and stress field may change in such a way that experimental residual stress values must be corrected. Different methods exist to account for the residual stress relaxation associated with the material removal operation and will be discussed in this paper.
\end{abstract}

\section{Introduction}

X-ray diffraction allows determining residuals stresses in surface. When we need to know stress gradients in depth, we have to use electropolishing layer removal. After this operation, material seeks a new equilibrium, stress fields change and then stress measurements must be corrected.

Strangely, few articles talk about the subject in the literature. The first one is the well-known Moore $\&$ Evans theory [1]. The second one is the Pedersen \& Hansson theory [2] and recently the SavariaBridier-Bocher theory [3].

In the following article, we will firstly expose a review of these different theories with a modification of the Pedersen-Hansson theory motivated by ours experiments. Then, we will compare theoretical/experimental results obtained on ladders. To finish, we will put side by side results obtained with the three different theories and results obtained thanks to the experiment.

\section{Review on residual stress relaxation associated with the material removal operation}

\subsection{Moore-Evans theory [2]}

Moore \& Evans' works were published in 1958. This method is the most used nowadays in the industry because it allows easy and fact results correction from X-ray diffraction method. Moreover, this method is recommended in the SAE HS-784 2003 standard. The theory is based on the following analytical formula which is easily computed:

$$
\sigma_{\mathrm{y}}(\mathrm{z} 1)=\ldots+2 \int_{z 1}^{H} \frac{\sigma d z}{z}-6 \mathrm{z} 1 \int_{z 1}^{H} \frac{\sigma d z}{z^{2}} .
$$

Where $\mathrm{z}$ is the thickness of the plate, and $\mathrm{z} 1$ the distance from the lower surface to the point of interest.

\subsection{Pedersen-Hansson theory and modification [2]}

a. Initial theory reminder

After Moore \& Evans work, two Danish researchers used Finite Element Method (FEM) at the end of the 80 's in order to simulate a material removal neither for an entire layer but only for a groove. Figure 1 shows a schematic drawing used for the FE simulation. It is a block of $200 \times 100 \times 100 \mathrm{~mm}^{3}$ with a symmetrical groove in the center. With the symmetry, it is considered only half of the block. The applied stress is considered as linear on the thickness. 


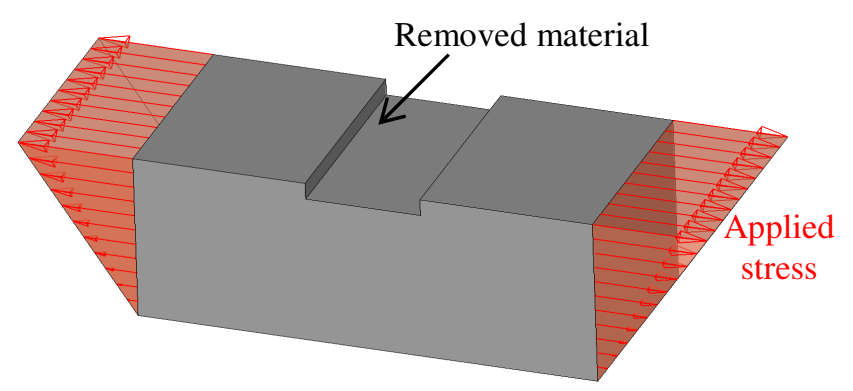

Fig.1 Schematic drawing of material removal in a block of 200x100x100 $\mathrm{mm}^{3}$.

Pedersen and Hansson compare the correction in per cent to add to the residual stress measurement in function of the material removal depth.

\section{b. Theory modification}

In our case, some changes have been made to take into account a more realistic geometry of material removal. Experimentally we use an electropolishing machine to remove a square or rectangle shape of few $\mathrm{cm}^{2}$ (between 1 and $5 \mathrm{~cm}^{2}$ ) of material. The X-ray analyses are always carried out at the center of the removed shape.

Then, concerning the electropolishing profile, Figure 2 illustrates the shape of the bottom of the groove considering an angle different to $90^{\circ}$. This is pertinent if the attack depth and the attack width are in the same units or same scales. In our case, it is not the case as presented in Figure 3. The attack width is roughly $15 \mathrm{~mm}$ whilst the attack depth is $100 \mu \mathrm{m}$. So, the depth attack is too tiny compare to the width attack to have an influence. The shape of the bottom for our model is considered with an angle equal to $90^{\circ}$.

In terms of boundary conditions, it has been decided to keep the same boundary conditions than Pedersen et al. which are linear stresses profile on the thickness from 0 to $+100 \mathrm{MPa}$.

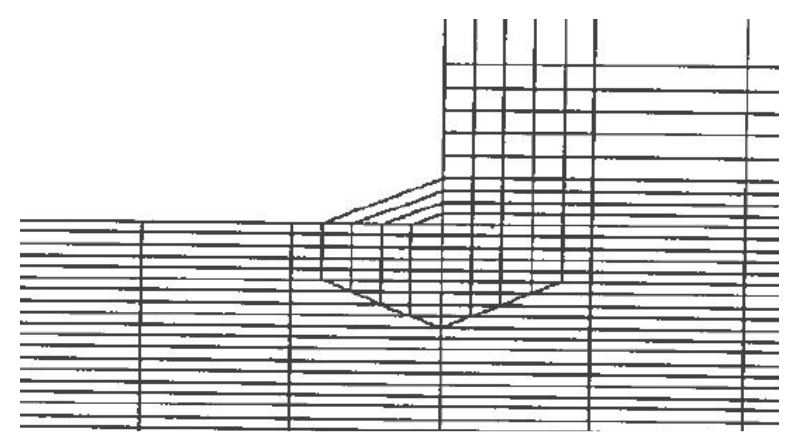

Fig. 2 Zoom at the bottom of the groove used by Pedersen et al. [2]

\subsection{Savaria-Bridier-Bocher theory [3]}

Savaria et al. published in June 2012 in the International Journal of Mechanical Sciences and developed a theory to take into account the stress relaxation in a tube.

In their theory, different calculation assumptions are used: Relaxation is elastic; no plasticity is introduced by residual stress relaxation/redistribution during removal layers.

- Removal layers technique do not introduced new residual stress.

- Residual stresses in the material are considered as uniforms on the entire specimen surface.

- Residual stresses in the material are considered as uniforms on the entire layer.

- Specimen and removal window geometry corresponding to material removal are the same for the simulation and the experimental part. 
- Stresses are uncoupled; it means that the relaxation and redistribution in one direction are only affected by previous stresses removed in that same direction.

As for the authors, the correction depends strictly on both factors below:

- the geometry (specimen and removed layer)

- the magnitude of previously removed stress in the direction of interest.

The authors claims that local stress variation observed at a depth $\mathbf{d}$ after a material removal step $\mathbf{s}$ is:

$$
\left(\Delta \sigma_{\mathrm{d}}\right)_{\mathrm{s}}=\left(\sigma_{\mathrm{d}}\right)_{\mathrm{s}}-\left(\sigma_{\mathrm{d}}\right)_{\mathrm{s}-1}=-\mathrm{K}_{\mathrm{ds}} \sigma_{\mathrm{ms}} \text {. }
$$

With $\mathrm{K}_{\mathrm{ds}}$ the correction coefficient at depth $\mathrm{d}$ associated to a material removal step $\mathrm{s}$ (this coefficient is not the same in all stresses directions and must be re-calculate regardless of each direction), $\left(\Delta \sigma_{\mathrm{d}}\right)_{\mathrm{s}}-\left(\Delta \sigma_{\mathrm{d}}\right)_{\mathrm{s}-1}$ are stresses in a direction at a depth $\mathrm{d}$ after material removals $\mathrm{s}$ and $\mathrm{s}-1$. $\sigma_{\mathrm{ms}}$ is the measured stress on the surface of the layer $\mathrm{s}$.

For all the others depths $\mathrm{d}$, corrected stress $\sigma_{\mathrm{cd}}$ are calculated using measured stresses $\sigma_{\mathrm{md}}$ for all the previous materials removed.

$$
\sigma_{\mathrm{cd}}=\sigma_{\mathrm{md}}+\sum_{s=1}^{d-1} K_{d s} * \sigma_{\mathrm{ms}} .
$$

We can write the previous equation (Eq. 3) in the form of matrix:

$$
\sigma_{\mathrm{c}}=[\mathrm{I}+\mathrm{K}] \sigma_{\mathrm{m}} .
$$

With $\sigma_{\mathrm{c}}$ and $\sigma_{\mathrm{m}}$ are columns matrices containing corrected and measured stresses components $\sigma_{\mathrm{cd}}$ et $\sigma_{\mathrm{md}}$ for depths from 1 to $\mathrm{n}$. I and $\mathrm{K}$ are respectively identity matrix and correction matrix (dimension n).

\section{Results and discussion}

\subsection{Four point bending test}

To evaluate the stress relaxation induced by material removal, it has been considered to load a specimen of $130 \times 15 \times 10 \mathrm{~mm}^{3}$. The sample in $\mathrm{S} 690$ has suffered a tempered at $550^{\circ} \mathrm{C}$ during 1 hour to remove the initial residual stresses.

The sample has been checked in the beginning of bending testing and considered as free stress sample (see Figure 5). The bending test device allows applying a load of $+550 \mathrm{MPa}$ on the surface monitored by a strain gauge localized in the opposite face of interest for X-ray analyses. So X-ray stress measurements are in compression. The measurement has been made in longitudinal direction of the sample. 


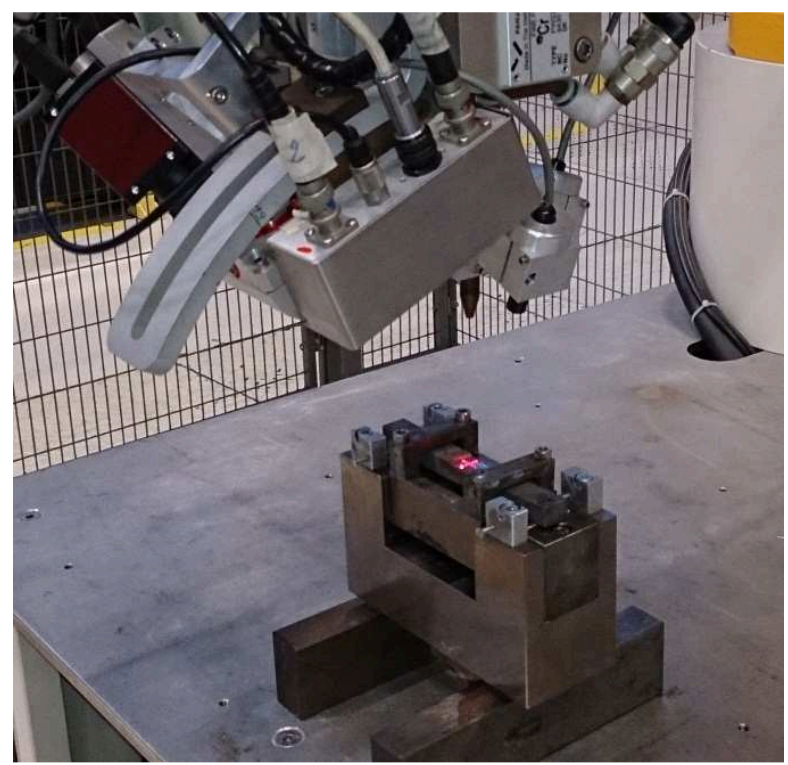

Fig. 5 X-ray analyses on bending bar.

Polishing with a hydrofluoric acid is made on the sample in bending for each removal layer with a metrological control. The sample stays in bending during all removal layer. At each material removal, stress analysis is carried out by X-ray diffraction. Table 1 presents the results of theoretical stress profile when $+550 \mathrm{MPa}$ are applied on a face of the sample in flexion in relation with the experimental results obtained by X-ray diffraction without correction and with the 3 methods presented above: Moore Evans, Pedersen-Hansson modified and Savaria et al. Figure 6 illustrates this table.

It may be noted that corrected profiles of Moore-Evans theory and Pedersen-Hansson modified are closed to the theoretical profile however the Savaria corrected profile do not present enough correction. These last results look strange because all assumptions and parameters developed by Savaria et al. are in agreement with the experimentation whilst Moore Evans and Pedersen-Hansson modified theories present more simplification.

Table 1 Comparison between theoretical/experimental/corrected profiles

\begin{tabular}{|c|c|c|c|c|c|}
\hline $\begin{array}{c}\text { Depth } \\
(\mathrm{mm})\end{array}$ & $\begin{array}{c}\text { Residual stress - } \\
\text { Theoretical } \\
\text { profile (MPa) }\end{array}$ & $\begin{array}{c}\text { Residual } \\
\text { stress - } \\
\text { Experimental } \\
\text { measurements } \\
(\mathrm{MPa})\end{array}$ & $\begin{array}{c}\text { Corrected } \\
\text { profile (Moore } \\
\text { Evans theory) }\end{array}$ & $\begin{array}{c}\text { Corrected } \\
\text { profile } \\
\text { (Pedersen- } \\
\text { Hansson } \\
\text { modified) }\end{array}$ & $\begin{array}{c}\text { Corrected } \\
\text { profile } \\
\text { (Savaria- } \\
\text { Bridier- } \\
\text { Bocher's } \\
\text { theory) }\end{array}$ \\
\hline 0 & -550 & -510 & -510 & -510 & -510 \\
\hline 0,07 & $-542,3$ & -502 & -492 & -488 & -508 \\
\hline 0,09 & $-540,1$ & -560 & -539 & -542 & -575 \\
\hline 0,22 & $-525,8$ & -531 & -490 & -484 & -537 \\
\hline 0,39 & $-507,1$ & -601 & -507 & -516 & -596 \\
\hline 0,58 & $-486,2$ & -599 & -457 & -470 & -573 \\
\hline 0,81 & $-460,9$ & -633 & -430 & -449 & -599 \\
\hline 1,02 & $-437,8$ & -648 & -385 & -412 & -587 \\
\hline
\end{tabular}




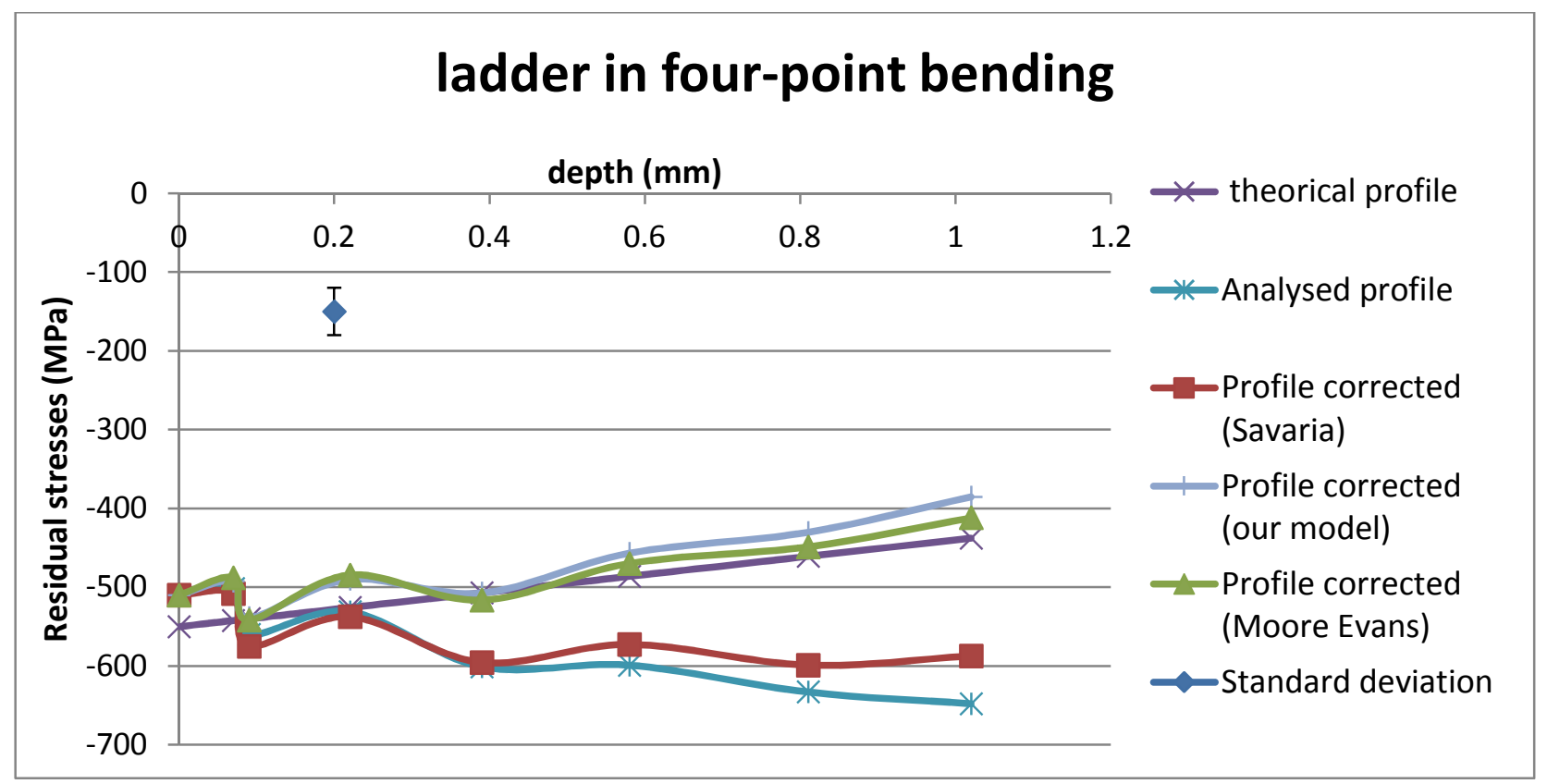

Fig. 6 Graphical comparison between theoretical/experimental/corrected profiles.

So it may be seen in Figure 6 that the correction of our model which means the modified PedersenHansson model may be validated.

\subsection{Influence of sample dimensions on the correction factor}

First of all, it is interesting to investigate the influence of sample dimensions on the correction factor.

Residual stress profile was obtained by X-ray diffraction with several electropolishing steps on a sample with different surface treatments. To evaluate the effect of removal layers on the initial profile, 6 geometry of specimen are considered :

- $\quad$ Block of 200x100x100 $\mathrm{mm}^{3}$

- $\quad$ Block of $200 \times 100 \times 50 \mathrm{~mm}^{3}$

- Block of $200 \times 50 \times 50 \mathrm{~mm}^{3}$

- Block of $200 \times 15 \times 5 \mathrm{~mm}^{3}$

- Sheet of $130 \times 50 \times 2.2 \mathrm{~mm}^{3}$

- Sheet of $130 \times 50 \times 1.8 \mathrm{~mm}^{3}$

- Sheet of $130 \times 50 \times 1.4 \mathrm{~mm}^{3}$

Figure 4 presents the initial profile without correction (analysed profile) and the 6 profiles in function of the geometry of the sample for block's correction. The corrections are provided by the modified Pederson et al. theory.

It may be seen that smaller and thinner is the sample, stronger is the correction which is also shown by Savaria et al. in their research [3].

Knowing that the real shape of analyzed sample looks like a big block of $200 \times 100 \times 50 \mathrm{~mm}^{3}$, the good profile should be used in consequence and in this case, material removal does not influence the stress gradient which means that the stress relaxation is not sensible to material removal. 


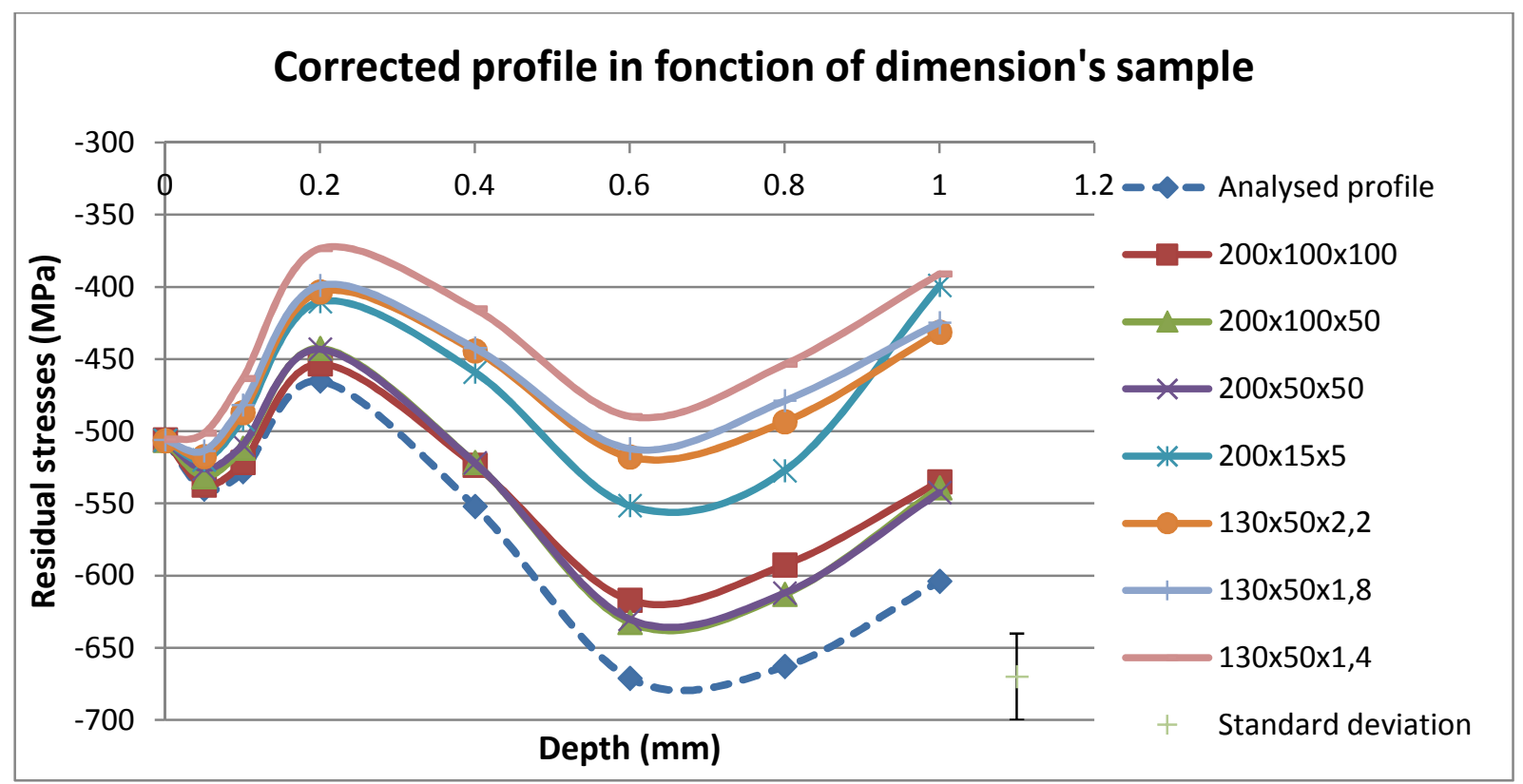

Fig.4 Influence of the sample's size.

\section{Conclusions}

It has been seen that the correction depends strongly on the size of the sample. The simulation must be as closed as possible to the real shape of the analyzed sample in terms of sample dimension and removal layer dimension.

Automatic program with multiple choices of basic geometry has been made to give easy correction profile to the technician in charge of residual stress analyses.

It seems that the Moore Evans or Pedersen-Hansson modified theories present the best results in comparison with the theoretical stress profile however Furthers investigations can be required to improve the model:

- Change the size of the sample.

- Increase or decrease the initial bending stress Consider service stresses as residual stresses but their behavior may be different.

- Compare the corrected profile with a profile made by synchrotron.

\section{References}

[1] Moore, M. and Evans, W., "Mathematical Correction for Stress in Removed Layers in X-Ray Diffraction Residual Stress Analysis," SAE Technical Paper 580035, 1958, doi:10.4271/580035.

[2] Pedersen TF, Hansson ILH." Finite element calculations for correction of residual stress profiles of coated and uncoated materials measured by X-ray diffraction." NDT Int 1989;22:347-52

[3] Savaria V., Bridier F. and Bocher, P., "Computational quantification and correction of the errors induced by layer removal for subsurface residual stress measurements", International Journal of Mechanical Sciences, Volume 64 (1) - Nov 1, 2012 